\title{
Integrative Properties of the Pel Neuron, a Unique Mushroom Body Output Neuron
}

Jürgen Rybak ${ }^{1}$ and Randolf Menzel ${ }^{2,3}$

${ }^{1}$ Department of Psychology

Dalhousie University

Halifax, Nova Scotia B3H 4J1, Canada

${ }^{2}$ Institut für Neurobiologie

Freie Universität Berlin

D-14195 Berlin, Germany

\section{Abstract}

A mushroom body extrinsic neuron, the Pe1 neuron, connects the peduncle of the mushroom body (MB) with two areas of the protocerebrum in the honeybee brain, the lateral protocerebral lobe (LPL) and the ring neuropil around the $\alpha$-lobe. Each side of the bee brain contains only one Pe1 neuron. Using a combination of intracellular recording and neuroanatomical techniques we analyzed its properties of integrative processing of the different sensory modalities. The Pe1 neuron responds to visual, mechanosensory, and olfactory stimuli. The responses are broadly tuned, consisting of a sustained increase of spike frequency to the onset and offset of light flashes, to horizontal and vertical movements of extended objects, to mechanical stimuli applied to the antennae or mouth parts, and to all olfactory stimuli tested (29 chemicals). These multisensory properties are reflected in its dendritic organization. Serial reconstructions of intracellularly stained Pe1 neurons using confocal microscopy reveal that the Pe1 neuron arborizes throughout all layers of MB peduncle with finger-like, vertically oriented dendrites. The peduncle of the MB is formed by the axons of Kenyon cells, whose dendritic inputs are organized in modality-specific subcompartments of the calyx region. The peduncular arborization indicates that the Pe1 neuron receives input

${ }^{3}$ Corresponding author. from Kenyon cells of all calycal subcompartments. Because the Pe1 neuron changes its odor responses transiently as a consequence of olfactory learning, we hypothesize that the multimodal response properties might have a role in memory consolidation and help to establish contextual references in the long-term trace.

\section{Introduction}

The mushroom bodies (MBs) are prominent brain structures in the central brain of insects. MBs are regarded as high order integrative centers of the insect brain. Their principal organization was described at the turn of the century (Kenyon 1896). Since then, there has been speculation about their functional role (Vowles 1955; Howse 1974, for review, see Erber et al. 1987; Menzel et al. 1994). Molecular analysis, electrophysiological recordings, elimination of MBs during ontogenetic development, and reversible interference of function with cooling probes showed that these properties have an important role in olfactory learning and memory processes, both in Drosophila and the honeybee (Heisenberg et al. 1985; Menzel 1990; Davis 1993; de Belle and Heisenberg 1994). The MBs in the honeybee are highly differentiated. They are located along the midline in the central brain, and each MB is formed by 170,000 intrinsic elements, the Kenyon cells (Witthöft 1967). Their dendritic arborizations give rise to concentrically arranged neuropils that form the median and lateral calyces. Each calyx is subdivided into lip, collar, dorsobasal, and basal ring subcompartments. Axonal projections of the Kenyon cells originating in the calycal zones project in bundles into the midbrain, thus forming the peduncle and the $\alpha$ -

LEARN IN G \& MEM ORY 5:133-145 @ 1998 by Cold Spring Harbor Laboratory Press ISSN 1072-0502/98 \$5.00

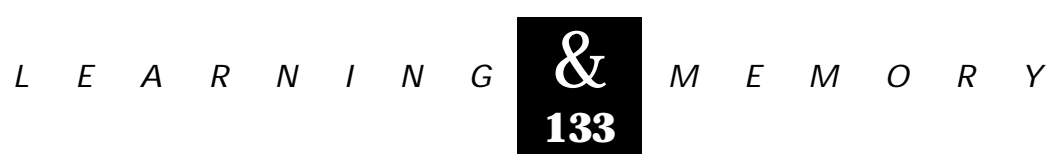


and $\beta$-lobes of the MB (Kenyon 1896; How se 1974; Mobbs 1982; Rybak 1994). Because of the highly ordered, parallel arrangement of the Kenyon cell axons, the MB in each brain hemisphere is partitioned into two subsystems: the median and lateral subcompartment. The calyces of the MB are modality-specific input stations. The lip and collar neuropils are innervated exclusively by antennal and visual tracts, respectively (Mobbs 1982; Menzel et al. 1994). The basal ring receives mixed input from various brain regions (Mobbs 1982; R. Abel, unpubl.). This compartmentalized organization of Kenyon cells is maintained within the peduncle and maps onto specific regions of the $\alpha$ - and $\beta$ lobes. Proto- and deutocerebral interneurons connect the axonal system of the MB at different levels along the peduncle and the lobes, with distinct areas in the protocerebral lobe and the antennal lobe (Schürmann 1974; Mobbs 1982; Rybak and Menzel 1993).

In the honeybee brain, interneurons associated with the MBs have been identified (Rybak and Menzel 1993). The pedunculus extrinsic Pel neuron changes its response properties to olfactory stimuli specifically when the animal is sensitized or conditioned to an odor (Mauelshagen 1993). The single Pel neuron in each MB represents a pair of large, identified neurons in the bee brain that form a major output pathway from the peduncle of the MBs (Mauelshagen 1993; Rybak 1994). The Pe1 neuron is positioned at a strategic site in the bee brain. It receives input from within the MB neuropil and transmits it to the protocerebrum, the lateral protocerebral lobe (LPL), and the ring neuropil around the $\alpha$-lobe. It also sends a few branches to the ventral $\alpha$-lobe, where it forms output synapses (Rybak and Mauelshagen 1994). The Pel neuron arborizes widely in the peduncle at a level where $\alpha$ - and $\beta$-lobes branch off. Because of the dendritic arbors span the whole cross section area of the peduncle we asked whether the Pel neuron might receive input from Kenyon cells of all calycal subcompartments, leading to multisensory convergence of Kenyon cells on a single, unique extrinsic neuron.

The question arises as to whether and how a multisensory integrating neuron like $\mathrm{Pel}$ might both serve the function of reading out specific (e.g., olfactory) memory and at the same time respond to a large variety of stimuli. We conceptualize that any form of associative learning needs to be related to other stimuli, thus setting the learning of a cue stimulus to the context in which it occurs.
To approach this question, we concentrate in an initial step on the study of the response properties of the Pel neuron and its dendritic arborization with the aim of setting the background for an analysis of the learning-induced changes in its multisensory properties. Convergence of multisensory input on an adaptive neuron may represent a neural strategy of encoding context-dependent plasticities. Before we can address this question, we need a better understanding of the structural and functional aspects of the multisensory properties of the Pel neuron.

\section{Materials and Methods}

\section{ANIMALS}

Honeybees (Apis mellifera carnica) were collected from the hive of the laboratory the day before they were used for electrophysiological recordings. Bees were fixed to a metal tube, and the heads were glued to a support spanning the neck region. Bees were kept overnight in a cool, dark, and moist compartment after having been fed with $2 \mathrm{~m}$ sucrose solution to satiation.

\section{ELECTROPHYSIOLOGY}

Conventional intracellular recording techniques were applied to sample membrane voltage fluctuations (synaptic potentials and action potentials) from Pel neurons in either isolated heads as performed by Mauelshagen (1993), or in whole animal preparations. The electrophysiological setup was the same as described by Mauelshagen (1993). Most results were gained by whole animal recordings, which in our experience allow more frequent data collection. For this, bees were fixed in a tube, as has been described previously for proboscis extension conditioning (Menzel et al. 1974; Menzel 1990). The head of the bee was glued to the tube by a drop of a wax-collophonium mixture, allowing the proboscis and antennae to move unhindered. A window was cut in the cuticle of the head capsule between the bases of the antennae, the median ocellus, and the inner borders of the compound eyes. The esophagus was pulled out through a second incision ventrally to the bases of the antennae by inserting a very fine forceps into the esophageal hole. A thin layer of vaseline was used to prevent dehydration of the brain. Animals in which the hemolymph level fell too low were discarded. Also, animals that showed strong brain

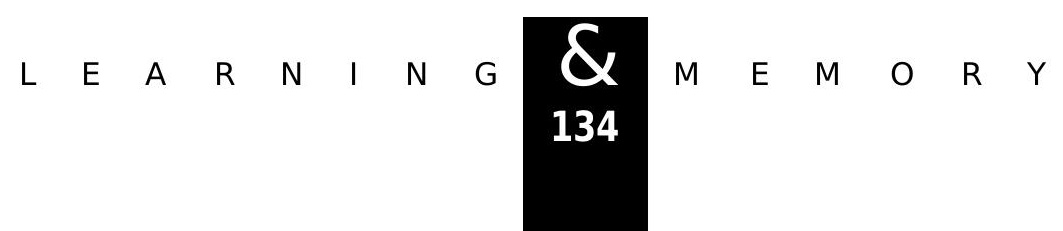


movements, caused by hemolymph pumping or muscle contractions, were not used. Recording electrodes (outer diam., $1 \mathrm{~mm}$ ) were pulled by a Flaming/Brown microelectrode puller (P87, Sutter Instruments). The resistance in the tissue ranged between 60 and $100 \mathrm{~m} \Omega$ for electrodes filled with $2.5 \mathrm{~m}$ potassium acetate and $140-180 \mathrm{~m} \Omega$ for electrodes filled with $4 \%$ lucifer yellow in $0.1 \mathrm{~m} \mathrm{LiCl}$. The Pel neuron was impaled at its neurite near the transition between peduncle and $\alpha$ - and $\beta$-lobe (ventrally to the $\beta$-exit point at a depth of 140-180 $\mu \mathrm{m} ;$ Mobbs 1982). The Pel neuron could be identified reliably with high probability during the process of recording because of its spike form and response properties (see Results). However, in about half of the recordings evaluated here ( 5 of a total of 11 recordings lasting longer than $15 \mathrm{~min}$ ), the recording site was confirmed by intracellular staining and subsequent morphological analysis. Neurons were iontophoretically filled with lucifer yellow by hyperpolarizing DC currents (2-4 nA) for several minutes. The brains were dissected under saline solution after 20-30 min diffusion time, thus allowing the dye to stain all parts of the neuron. The specimens were fixed in $4 \%$ paraformaldehyde in Millonig buffer ( $\mathrm{pH}$ 7.3), dehydrated in an ascending ethanol series, and cleared in methylsalicylate. The whole-mount preparations were viewed under epifluorescence and analyzed with the confocal microscope (Leica). Reconstructions were made from a series of optical sections at the confocal microscope.

\section{STIMULATION AND DATA EVALUATION}

Twenty-two different odor compounds were tested in all preparations (see Fig. 3, below); 7 additional stimuli consisting of mixtures of citral, 2hexanol, benzaldehyde in different combinations were used in only three preparations. Olfactory stimuli were provided either by directing the tip of a 20-ml plastic syringe containing a piece of filter paper soaked with the undiluted odorant toward the antennae and blowing its content over the head or by moving a glass vessel containing the undiluted compound (or mixture) into the pathway of a continuos slow airstream. In the first case, a sharp onset of the odor stimulus and a lasting stimulation with a rather constant odor stream was acquired, but the odor stimulation was also combined with a slight mechanical stimulation (Fig. $2 a, b$, below). In the latter case the odorant was added to a constant airstream produced by an ex- haustion piper (diam., $10 \mathrm{~cm}$ ) that was mounted behind the preparation. This procedure avoided the additional mechanical stimulus, but it did not allow the precise determination of the onset, duration, and concentration of the compounds, because the odor plumes were carried across the antennae not in a constant stream but in eddies. How ever, this stimulation technique allow ed the testing of a large number of chemicals in quick succession (one stimulus of 5-7 sec duration every $10 \mathrm{sec}$ ) during the recording of the Pel neuron. The time point when an odor eddy reached the antennae was indicated by a clearly detectable phasic $\mathrm{ON}$ excitation of the Pel neuron (Fig. 2c,b, below). Mechanical stimuli were applied to the antennae, mouth parts, and the dorsal region of the head between the compound eyes using a tiny brush with only a few hairs attached.

Visual light on-off stimuli were produced by switching the illumination of the dissection microscope on and off, which focused the light via two light guides onto the dorsofrontal parts of both compound eyes. Moving stripes were presented to the left compound eyes using a handheld $10 \times 10$ $\mathrm{cm}$-wide screen illuminated from behind. In front of the screen, a grating of black-and-white bars moved from left to right or in the opposite direction. The screen appeared at a visual angle of $90^{\circ}$, and the spatial wavelength of the grating was $9^{\circ}$. Depending on the orientation, the screen was moved front to back, back to front, or up- and downward. Sucrose stimuli were applied to the antennae (ipsi- or contralateral to the recording site) by slightly touching the flagellum with a wooden toothpick soaked in $2 \mathrm{~m}$ sucrose solution. To stimulate the glossa of the proboscis selectively, only the middle part of the toothpick was soaked. By gently moving the dry tip of the toothpick under the proboscis and advancing sidew ays slightly, the glossa was maneuvered to the sucrose part of the toothpick. Combined stimuli were tested for odor-mechanical antennal stimulation. The antennae were touched gently with a piece of filter paper from a used odor syringe.

All intracellular recordings were stored by an FM tape recorder and later recorded on a Brush pen w riter. Frequencies of action potentials (APs) were evaluated by counting the number of APs in 0.5 -sec intervals. The analysis indicated that the responses to odorants were rather variable. Because of the stimulation procedure it was not possible to separate clearly stimulus variability from response variability. Because the Pel neuron did

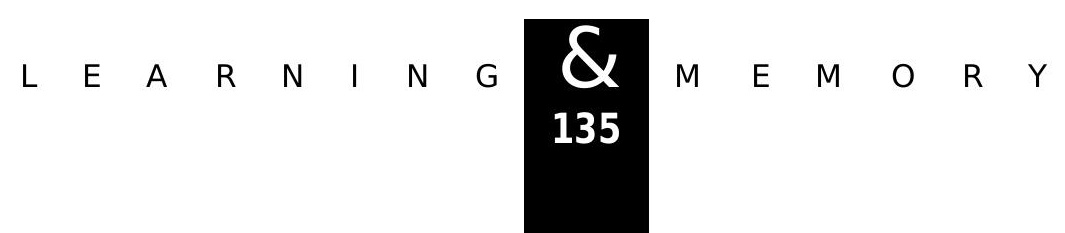


not give any indication of odor-specific responses and because the aim of this study was to test as many different stimuli as possible, we also categorized the responses of the Pel neuron with respect to the AP frequency using an audio monitor and distinguished three categories-no response, weak response ( $20 \%-50 \%$ increase of spontaneous activity), and strong responses (approximately $>50 \%$ increase of spontaneous activity). These categories were used only to detect any strong deviations from the general broad response profile to a large number of odorants and to decide during the experiment whether additional stimulations were needed to clarify the response profile.

\section{HISTOLOGY}

Bodian staining was used according to the protocol from Gregory's modified Bodian protargol technique (Clark 1973). Briefly, brains were fixed for 3-4 hr in AAF, a mixture of acetic acid, ethanol, and formalaldehyde (Blest 1961), dehydrated in an ascending ethanol series, cleared in xylene, embedded in paraffin, and sectioned at $12 \mu \mathrm{m}$ thickness. Sectioned material was deparaffinized in xylene and hydrated in a descending ethanol series. Staining was performed with Protargol S (Laboratoire Roques, France). A mixture of hydroquinone and sodium sulfite was used for reduction of silver. In the final steps, the sections were toned with gold chloride, developed in oxalic acid, and fixed with sodium thiosulfate. After dehydrating, the sections were mounted in Entellan (Merck, Germany). The staining cycle was repeated to enhance staining. For Golgi preparations the brains were incubated using a modified Golgi-Collonier technique (Strausfeld 1980). They were immersed in a Karnow sky fixative containing $2 \%$ paraformaldehyde/ $2 \%$ glutaraldehyde in sodium cacodylate buffer $(\mathrm{pH}$ 7.4) followed by a wash in the same buffer. The osmolarity of the fixative was adjusted by adding sucrose. The chromation was done in a mixture of one part $2 \%$ glutaraldehyde and four parts $4 \%$ potassium dichromate for 4-5 days in vials placed in the dark at room temperature. Following several washes in Holmes Borax-Borat buffer (pH 7.4) (Nässel and Seyan, cited in Strausfeld 1980) the specimens were washed several times in $0.1 \%$ aqueous silver nitrate solution until no more precipitates were formed. The final silver impregnation was done in $0.75 \%$ silver nitrate. Preparations were viewed as whole mounts under the microscope.

\section{IMAGING}

Images were obtained with a confocal microscope (Leica, Germany) using $16 \times$ and $40 \times$ primary magnification. Whole-mount preparations of the Pel neuron, intracellularly filled with lucifer yellow, were scanned in a frontal view. Reconstructions were made from printouts of $0.5-\mu \mathrm{m}$ thick optical sections.

\section{Results}

\section{PHYSIOLOGY}

GENERAL PHYSIOLOGICAL CHARACTERISTICS

As reported by Mauelshagen (1993), the Pe1 neuron (Fig. 1) can be recognized reliably by its physiological features, which are clearly distinguishable from neurons running in the anterodorsal protocerebral commissure [a.d.p.c. neurons (Mobbs 1982; Homberg 1984)] or A7 neurons (Rybak and Menzel 1993) whose neurites also leave the MB close to the Pel neuron at the transition of the peduncle to the lobes, the so called $\beta$ exit (Mobbs 1982). Summating excitatory postsynaptic potentials (EPSPs) are recorded during spontaneous activity and occur more frequently during stimulation (Fig. 2). APs (20-40 mV) ride on top of the EPSPs, but the variable temporal relationship between EPSPs and AP occurrence indicates that APs are conducted passively over some distance from the spike-initiating zone. Spontaneous AP activity with frequencies of $5-30 \mathrm{~Hz}$ occurs rather irregularly; how ever, in some cases, bursts of two or three spikes with regular interburst intervals occur. The intervals between the short bursts range between 150 and $250 \mathrm{msec}$, and AP frequency during the burst is $\sim 60-200 \mathrm{~Hz}$. The change from an irregular to a bursting pattern is abrupt and occurs un redictably from time to time, confirming observations by Mauelshagen (1993); this change was not found to be correlated with any obvious external stimuli (e.g., low level of stimulation by chemical, mechanosensory, or optical stimuli) or behavioral parameters (antennal position or movement, movement of the mouth parts). When the spontaneous AP activity was high ( $>20 \mathrm{~Hz})$, no grouping of APs in bursts was recorded. Slight hyperpolarization of the membrane potential ( -3 to $-7 \mathrm{mV}$ below resting potential of -35 to $-45 \mathrm{mV}$ ) reduced the AP frequency, with spikes occurring typically in bursts of two or three spikes (in 3 of the 11 preparations). These characteristic patterns allow

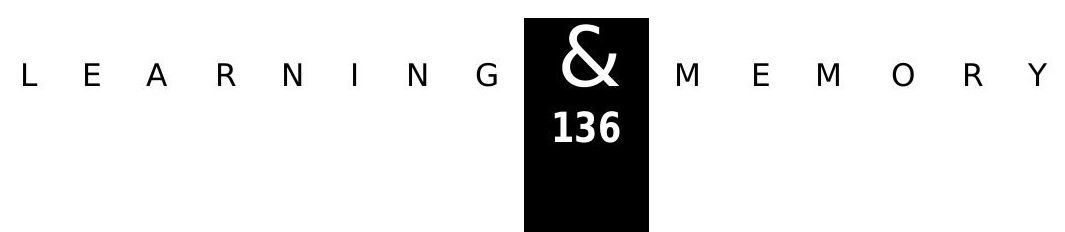



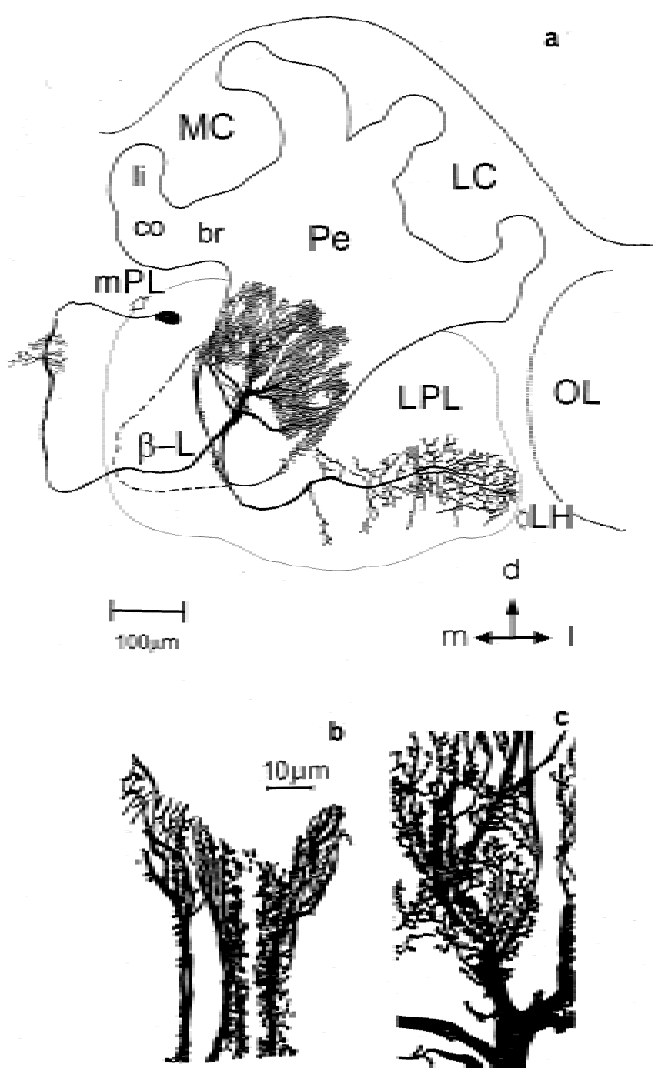

Figure 1: (a) Reconstruction of the Pel neuron within the protocerebrum of the honeybee from a cell filled intracellularly with lucifer yellow. The Pel neuron enters the $M B$ at the border from the $\beta$-lobe $(\beta-L)$ to the peduncle $(\mathrm{Pe})$ and arborizes extensively in the peduncle. The soma is located below the anterior brain surface and gives rise to a neurite that arborizes in the median contralateral protocerebral lobe ( $\mathrm{mPL})$. In the protocerebral lobe one branch courses lateral, gives rise to arborizations in the lateral protocerebral lobe (LPL), and terminates in the lateral horn (LH). (MC) Median calyx; (LC) lateral calyx; (li) lip; (co) collar; (br) basal ring; (O L) optic lobe. Scale, $100 \mu \mathrm{m}$. (d) Dorsal; (I) lateral; (m) median. $(b, c)$ Magnified parts of the Pel neuron in the posterior (b) and more anterior peduncle (c). Note the spiny processes along the course of the dendrites. Scale in $b$ and c, $10 \mu \mathrm{m}$.

the identification of the Pel neuron based on physiological parameters, because no other neuron recorded and stained in the $\beta$-exit region showed similar properties.

\section{OLFACTORY RESPONSES}

Responses to lasting odor stimuli ( $>2 \mathrm{sec}$ ) are typically phasic-tonic with onset frequencies in the range of $100-300 \mathrm{~Hz}$ and sustained responses in the range of $50-100 \mathrm{~Hz}$. The responses to odor pulses of $1 \mathrm{sec}$ give only the phasic component (Fig. 2a,b), and responses to the stimulation procedure applied most frequently in our study (presentation of a glass vessel containing the undiluted odorant into the pathway of a continuous airstream) indicate the arrival of odor jetties at irregular intervals, differing in concentration and duration. Such a stimulation allows testing of a large number of compounds in quick succession, and in addition, it resembles more closely the natural form of odor stimulation. In the natural environment, odor eddies reach the antennae in packages of varying frequencies. The frequency of odor packages might be indicative of the distance to the odor source, as air turbulence separates the packages with increasing distance. It was, therefore, interesting to see whether the Pel neuron can monitor such temporal structures. Figure 2, c-e, shows that the responses to such stimuli are highly structured, indicating a sensitivity to the sequential arrival of odor packages and a capacity to code the sequential arrival of odor eddies at a time scale well below $1 \mathrm{~Hz}$. Different forms of complex spiking

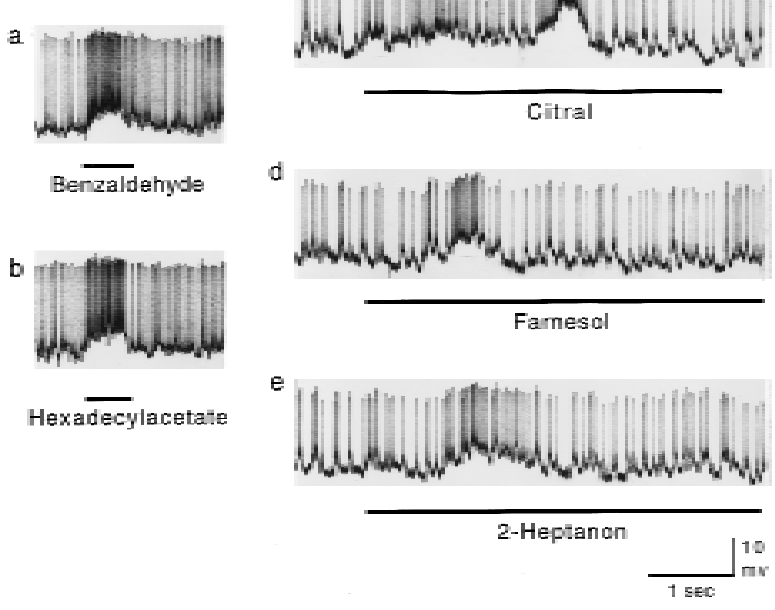

Figure 2: Examples of Pel responses to different chemical compounds (a) benzaldehyde; $(b)$ hexadecylacetate; (c) citral; (d) farnesol; (e) 2-heptanone. Stimulation in $a$ and $b$ was $1 \mathrm{sec}$ using a syringe for fast stimulus onset; in $c-e$, a glass vessel containing the undiluted odorant was brought into a constant airstream (see $M$ aterials and M ethods). Because of the latter stimulation procedure, stimulus onset is rather gradual, and both stimulus strength and duration may vary. Fluctuations in spike activity as seen in c-e may thus indicate eddies of odorants. 
patterns were elicited by step-like odor stimulation (see Fig. 4D in Mauelshagen 1993) indicating that long-lasting and constant odor stimulation induces additional intrinsic response dynamics. It has yet to be analyzed how these different forms of temporal dynamics interact, for example, whether the Pel neuron is tuned to particular stimulus frequencies.

Twenty-nine different odorants were tested. Figure 3 shows a representative example for a recording, where 22 odorants were applied. As in all other cases, this Pel neuron responds with excitation to all odors. Strong responses are found for the aldehydes benzaldehyde and hexanal, the alcohol 2-hexanol, the ketone pentanon, the acetate hexadecylacetate, and the terpen citral. In 11 Pel recordings we did not find an odorant that was not responded to with at least a weak increase in AP frequency. Other odor stimuli included mixtures of citral, 2-hexanol, and benzaldehyde. No differences in the AP patterns to the tested compounds were found. This applies also to the mixtures tested. The mixing of tw o components leads sometimes to an increase of the response, but in most cases no difference was seen when compared to the response to the most effective single component. It is therefore likely that the concentration dependence, which we did not test specifically, may have reached response saturation of the Pel neuron or at any stage upstream of Pel (see also below). Repetitions of stimulation indicated that the differences between strong and weak responses were odor specific, but the variability between different neurons and from stimulation to stimulation of the same neuron was rather large. That the variability of responses is at least partially due to the stimulation procedure cannot therefore be excluded.

\section{RESPONSES TO OTHER SENSORY MODALITIES}

Contact chemoreceptive, mechanosensory, and visual stimuli evoke excitatory responses in the Pel neuron as well (Fig. 4). Mechanical stimulation at one or both antennae and proboscis including the glossa (but not at other locations on the head) induce phasic ON responses (Fig. 4c). The same is true for sucrose stimulation of the antennae or glossa. Both stationary visual stimuli (ON, OFF) and moving stripes lead to phasic excitatory responses. The response to light OFF is stronger than that to light ON (Fig. 4a). In both test situations adaptation time was about equal (several minutes light or dark). Motion stimuli are effective but are not discriminated: All four directions tested (front to back, back to front, up-, and downward) elicit similar response patterns (Fig. 4b). We tested also stimulus combinations, for example, an odor (citral or 2-hexanol) combined with mechanical stimulation to the antennae. The responses were always stronger than to the mechanical stimulus alone. How ever, the variability in the response did not allow us to determine whether the stronger response was just the linear addition of the individual responses or more. At least this result indicates that the failure to detect an additive effect in odor mixtures does not result from a saturated re-

Figure 3: Pel responses to 23 different pure compounds of five groups of chemicals. The animal was stimulated with a glass vessel introduced into an airstream carrying odor plumes across the antennae. This stimulation leads to responses as shown in Fig. 2, c-e. Spike frequency was evaluated for the strongest response component during a 5 -seclong stimulation indicative for the arrival of an eddy of highest odor concentration. The ordinate gives the change of spike frequency relative to spontaneous frequency $(100 \%)$. Aldehydes: (Benz) benzaldehyde; (Hex) hexanal; (Hept) heptanal; (2-O ct) 2-octanal. Alcohols: (2-Hex) 2-hexanol; (2-H ept) 2-heptanol; (Hexadec) hexadecanol; (2-O ct) 2-octanol; (tri-D ec) tri-decanol. Ketones: (2-H ex) 2 -
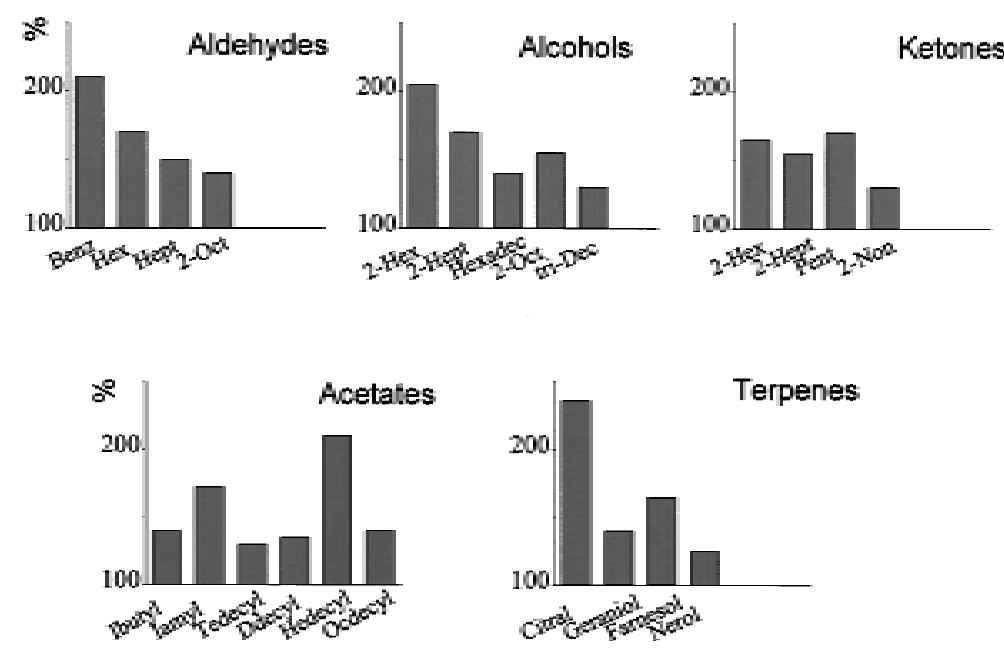
hexanon;(2-Hept) 2-heptanon; (Pent) pentanon; (2-N on) 2-nonanon. Acetates: (Ibutyl) isobutylacetate; (lamyl) isoamylacetate; (Tedecyl) tetradecylacetate; (D decyl) dodecylacetate; (Hedecyl) hexadecylacetate; (O cdecyl) octadecylacetate. Terpenes: As indicated.

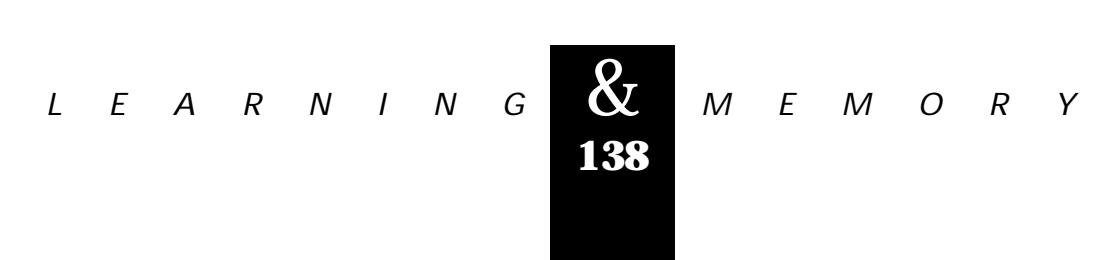


PE1 NEURON IN THE BEE BRAIN

a

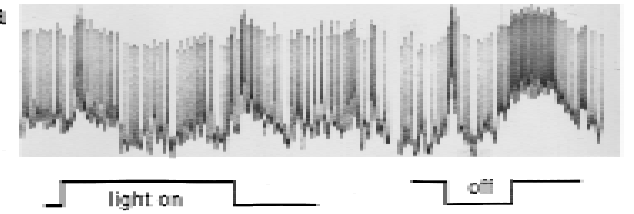

b

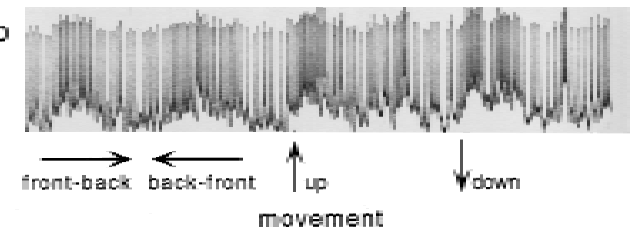

c.

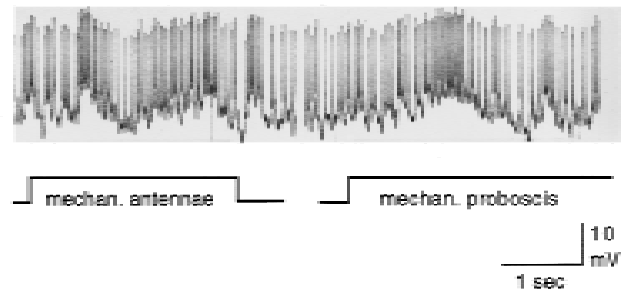

Figure 4: Pel responses to different modalities: (a) Light ON , light OFF; (b) moving stripes; (c) mechanical stimuli to antennae and proboscis.

sponse of the Pel but might indicate response saturation in the olfactory pathway upstream of Pel.

It thus appears that the Pel neuron integrates not only olfactory stimuli but also contact chemosensory, mechanosensory, and visual stimuli. We may therefore predict that in principle, the Pel neuron is synaptically connected with Kenyon cell subpopulations that derive from all modality-specific calycal subcompartments.

\section{MORPHOLOGY}

If one of the basic characteristics of the Pel neuron would be to integrate information from different sensory modalities, this should be implemented in its morphological organization within the Kenyon cell bundles arranged in parallel in the peduncle. To test this hypothesis we studied the arborization pattern of the Pel neuron within the peduncle [for an account of Pel synaptic connectivity on the electron microscopy (EM) level, see Rybak and Mauelshagen 1994]. To allocate the projections of the Pel neuron in the peduncle to calycal subcompartments and reveal its structure at the light microscopy level, we performed a confocal analysis of lucifer yellow-filled cells. Additionally, we compared the results of Bodian- and Golgistained preparations (Fig. 5a,b).
INTRINSIC PATTERN OF THE MBS

The complex pattern of Kenyon cell axonal projection in the MB stems from the fact that bundles of axons deriving from different calycal zones split off at different depths throughout the peduncle (see below) and form bands in the $\alpha$-and $\beta$-lobes. This structural organization is described as the transformation of circular coordinates (dendritic arborizations of Kenyon cells in the calyces) into the Cartesian coordinate of the peduncle and lobes (Howse 1974; Mobbs 1982). The analysis of the matching of intrinsic and extrinsic neuronal elements is facilitated by the fact that Kenyon cell axon bundles are organized in a highly ordered fashion without the crossing of fibers. Also, because each MB consists of two parts that are separated anatomically at the level of the calyx (median and lateral calyx; Fig. 1), but otherwise equally built (Rybak 1994), the same topographical principle applies to axonal projections originating in the median and lateral calyx. Thus, if the Pel neuron arborizes across the peduncle it should sample a dual set of rather similar information coming from the two calyces.

Proximal to the calyx, axon bundles of the Kenyon cells form a three-layered proximal peduncle ( $p$-Pe in the median calyx in Fig. 5a). More ventrally, where the most distal dendrites of the Pel neuron are found, the pedunculi of both calyces fuse and Kenyon cell axons begin to split. In this part of the peduncle, which we name distal peduncle (d-Pe in Fig. 5a), groups of axon bundles with different directions can be distinguished. Longitudinal strands project medioventrally to form the $\beta$-lobe; axons grouped in vertically oriented, finger-like zones run anteriorly to build up the $\alpha$ lobe (arrows in Fig. 5a). This organization is the consequence of the splitting of axons into two collaterals projecting into the $\alpha$-lobe and $\beta$-lobe, respectively (Fig. 5b). The $\alpha$ - and $\beta$-lobes are defined here as neuropils, in which all of the axon collaterals that are separated in the distal peduncle are fused to form the relative homogenous texture of the lobes. For the $\alpha$-lobe, this is a cylinder that extends from the anterior brain surface to a depth of $\sim 200 \mu \mathrm{m}$.

ZONE ALLOCATION IN THE PEDUNCLE AND LOBES TO CALYX SUBCOMPARTMENTS

The ordered arrangement of parallel-running axons allows the allocation of zones in the pe-

\section{$\begin{array}{lllllllllllllll}L & E & A & R & N & \text { I } & N & G & \underset{\mathbf{1 3 9}}{\mathbf{X}} & M & E & M & O & R & Y\end{array}$}



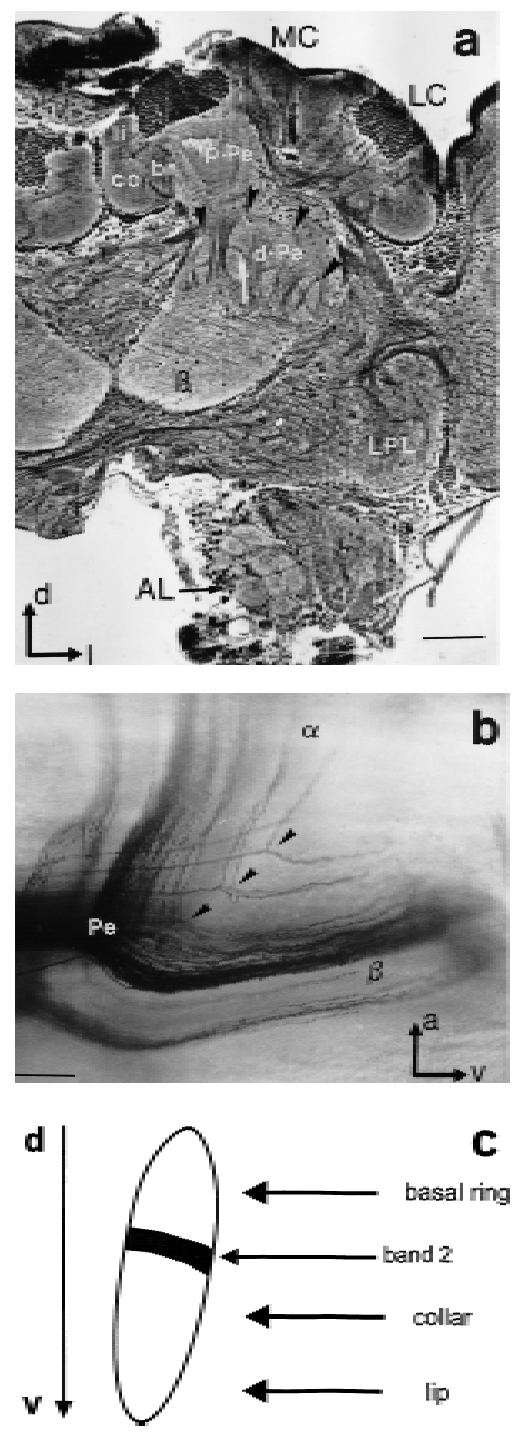

Figure 5: Structural organization of the MBs and Pel innervation. (a) Bodian staining reveals subcompartments of MBs at a depth of $\sim 250 \mu$ m (frontal view). The calyces (MC, LC) are composed of ring neuropils, lip, collar, and basal ring (li, Co, br). Axonal projections of the Kenyon cells form the layered proximal peduncle (p-Pe). Axons in the distal peduncle (d-Pe) split into collaterals more distal to the calyces. The finger-like structures (black arrowheads) are collaterals that form more anteriorly the $\alpha$-lobe, which extends into more frontal sections. Longitudinal areas extend into the $\beta$-lobe $(\beta)$. $N$ ote the banding pattern in the vertical structures (arrow). ( $A L$ ) Antennal lobe; ( $\mathrm{LPL}$ ) lateral protocerebral lobe. Scale, $100 \mu \mathrm{m}$. (d) Dorsal; (I) lateral. (b) Sagittal view of a $\mathrm{G}$ olgi-Collonier staining of Kenyon cell axon bundles at the splitting point [transition from peduncle (P) to $\alpha$ - and $\beta$-lobe, $\alpha$ and $\beta$ ]. Scale, $50 \mu \mathrm{m}$. (a) Anterior; (v) ventral. (c) Allocation of calycal subcompartments (basal ring, collar, and lip) to zones in the peduncle. The black bar in the finger-like structure represents the thin band shown in a (arrow). duncle and lobes to calycal zones. The distal peduncle (d-Pe in Fig. 5a) extends from the fusion of both pedunculi at a depth of $400 \mu \mathrm{m}$ and ends with the base of the $\alpha$-lobe $\sim 200 \mu \mathrm{m}$ depth. The specific texture caused by the intermingling and dividing axons is clearly distinguishable from the layered organization in the proximal peduncle and the lobes as seen in Bodian preparations (Fig. 5a), as well as in preparations with intracellularly labeled cells (Fig. 6). Throughout the peduncle Kenyon cell axons from all calycal subcompartments are split in a sequential order; most posteriorly Kenyon cell axons from the basal ring subcompartment divide and send collaterals to the dorsal $\alpha$-lobe and posterior $\beta$-lobe. Kenyon cells from the lip subcompartment split more anteriorly (close to the $\alpha$-lobe) and send fibers into the ventral $\alpha$-lobe and anterior $\beta$-lobe (see arrows in Fig. 5b). Collaterals that project tow ard the $\alpha$-lobe are visible as fingerlike structures in the distal peduncle (Fig. 5a). These vertically oriented zones begin to form posteriorly and become gradually larger until all axons are split. The pattern that finally forms the $\alpha$-lobe is seen most clearly at a depth of $\sim 200 \mu \mathrm{m}$, at the tripartition of lobes and distal peduncle. Thus, the stratifications at this level can be allocated to the calycal zones (Fig. 5c) and can be interpreted as a preformation of the $\alpha$-lobe.

\section{ARBORIZATION OF THE PEI NEURON} WITHIN THE PEDUNCLE

How do the arborizations of the Pel neuron fit into the scheme described above? The Pel neuron enters the MB neuropil with a large diameter neurite $(12-15 \mu \mathrm{m})$ at the tripartition of peduncle and lobes (Figs. $1 \mathrm{a}$ and $6 \mathrm{a}$ ). Its entrance point lies at a depth of $180 \mu \mathrm{m}$. The neurite splits off and gives rise to finger-like ramifications within the peduncle (Figs. $1 a$ and 6 ). The fine arborizations bear spinelike processes (Figs. 1, b and c, and $6 \mathrm{~d}$ ), indicating postsynaptic structures. Pel arborizations in the peduncle cover a large area ranging anteriorly from the base of the $\alpha$-lobe $(\sim 200 \mu \mathrm{m}$ depth) to the beginning of the proximal peduncle $(\sim 400 \mu \mathrm{m}$ depth). Comparison of the regions innervated by the Pel neuron as revealed with Bodian stain (Fig. 5a) indicates that Pel arborizations cover the whole distal peduncle, projecting more anteriorly within the part of the peduncle that comprises axons of Kenyon cells originating in the lateral calyx. This corresponds to the fact that in the lateral calyx axons begin to split off more posteriorly. The Pel dendrites do not enter the proximal peduncle

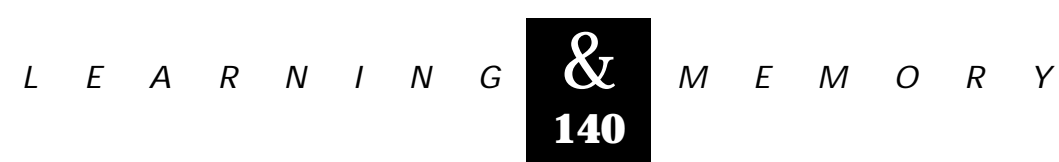


PE1 NEURON IN THE BEE BRAIN
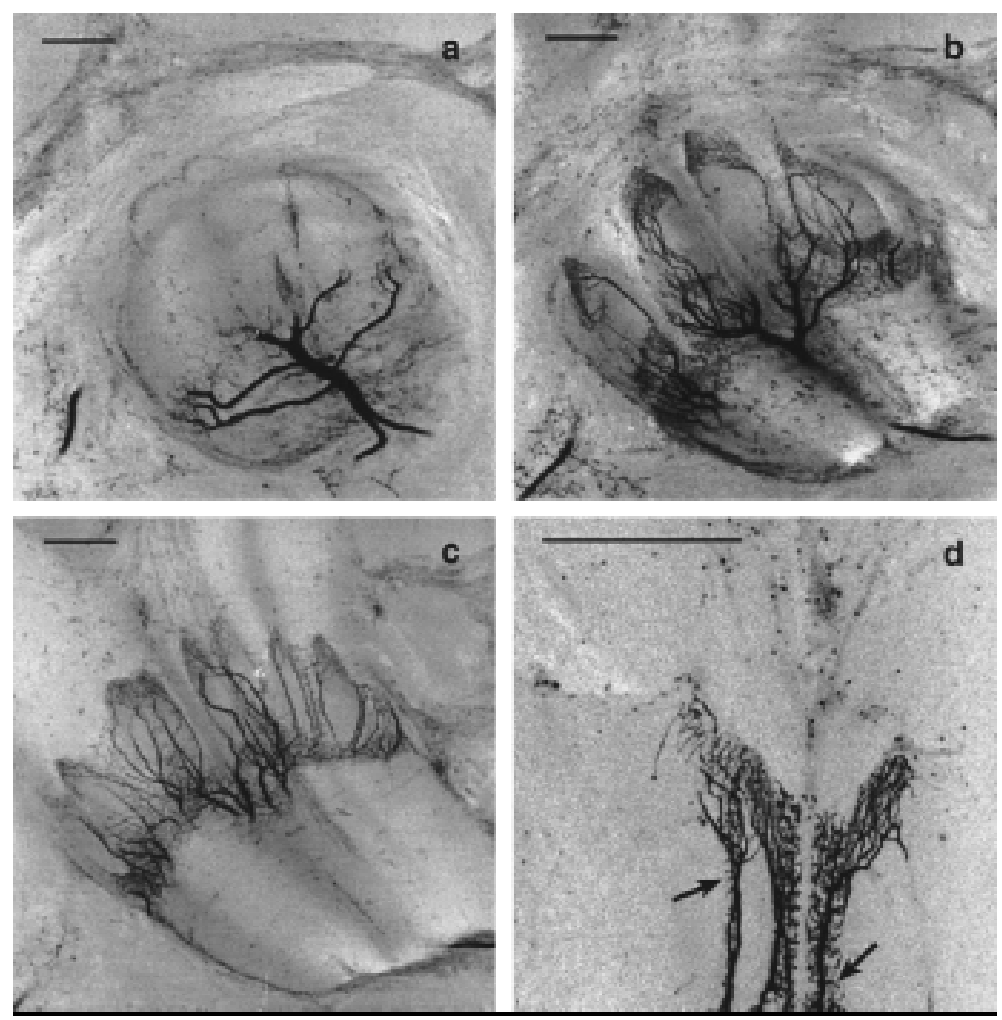

Figure 6: $\quad(a-c)$ Confocal images at different depth of the peduncle. The main dendrite enters the $M B$ at the transition of the peduncle to $\alpha$ - and $\beta$-lobe, and its fine arborizations are restricted to vertically oriented zones. Compare with Fig. 5a. Scale, $50 \mu \mathrm{m}$. (d) Fine structure of the Pel neuron in the ventral peduncle at a higher resolution. Note the spiny endings (arrows). Scale, $50 \mu \mathrm{m}$. or the lobes (but see below for fine axon projections of the Pe-1 to the $\alpha$-lobe). Because the Pel neuron arborizes exclusively in vertical areas of the "splitting zone" of the distal peduncle, it contacts primarily Kenyon cell collaterals that form the $\alpha$ lobe. The collaterals of the Pe1 neuron form narrow bands in different depths of the peduncle and show spiny processes along the course of thicker axons. Thus, the Pel neuron is connected with Kenyon cell projections stemming from all calycal subcompartments and is therefore anatomically w ell suited for integrating sensory information present in the MB system.

\section{PEI ARBORIZATIONS IN THE PROTOCEREBRAL LOBE}

Outside the MB, the Pel neuron has extensive arborizations in the protocerebral lobe (Figs. 1 and $6)$. It sends branches in the ring neuropil around the $\alpha$-lobe and the LPL, where they arborize extensively in the lateral horn (LPL and LH in Fig.1a). The soma (diam., $30 \mu \mathrm{m}$ ) gives rise to the primary neurite that courses contralaterally into the medial protocerebral lobe (mPL) and posterior. The neurite describes a posteriorly directed loop through the $\mathrm{mPL}$, where it sends off small branches (Fig. 1a). In a depth of $\sim 210 \mu \mathrm{m}$ from the anterior brain surface the primary neurite crosses back ipsilaterally to the MB. A major branch enters the MB at the $\beta$ exit as described above (Fig. 1a).

Ventrally to the $\beta$-exit in the protocerebral lobe, the main neurite gives rise to a second large process that follows the ventrolateral margin of the $\alpha$-lobe. Here lies the origin of several branches that invade the ring neuropil around the $\alpha$-lobe. Originating from processes in the ring neuropil, one median and one lateral branch innervate in a depth of $100-120 \mu \mathrm{m}$ the ventral $\alpha$-lobe with fine and also bleb-like varicosities (not shown in Fig. 1a). This region was described as the $A 4$ region and is assumed to carry preferentially olfactory information (Rybak and Menzel 1993; Rybak 1994, see also Fig. 7). The $\beta$-lobe is not innervated by Pel profiles.

At the lateral margin of the $\alpha$-lobe one large axon turns lateroventrally and posteriorly into the LPL to terminate with bleb-like endings in two large projection areas at a depth ranging between 30 and $300 \mu \mathrm{m}$ (Fig. 1a). One of them is located more anteriorly, and the other directed posteriorly, thus spanning the whole area of the so-called LH. This area is known as a projection area of antennocerebralis tracts (ACTs) originating in the anten- 


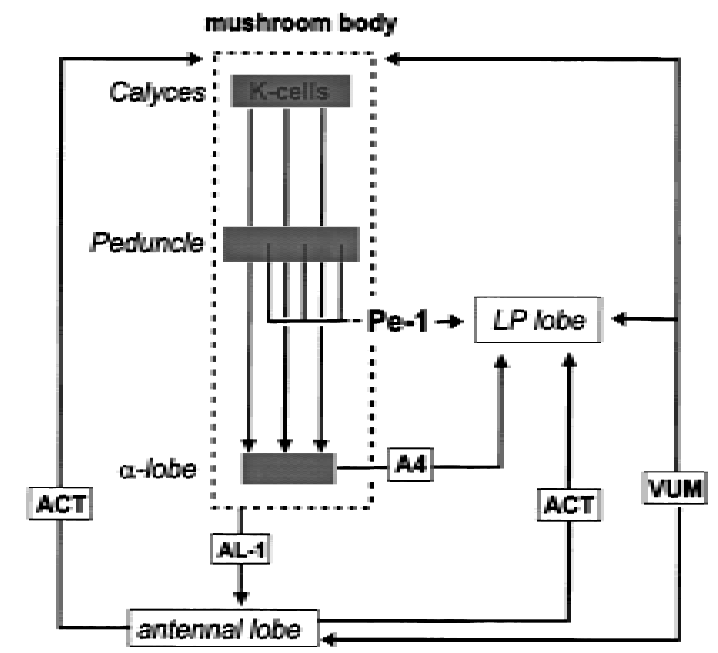

Figure 7: Summary of Pel neuron projections and other neuronal pathways involved in olfactory processing and learning in the honeybee brain. The Kenyon cells of the MB project onto the extrinsic Pel neuron in the peduncle. Outside the MB the Pel neuron projects to premotor centers of the protocerebrum (LP lobe), which includes the LH. In the LP lobe the Pel neuron converges with the VU Mmx1 neuron (VUM), which also projects to the antennal lobe and the calyces. Furthermore, Pel overlaps with the A4 cells (output neurons of the $\alpha$-lobe), and the AL-1, a feedback neuron from the MB to the antennal lobe. (K-cells) Kenyon cells; (ACT) antennocalycal tract. (Adapted from Rybak and Menzel 1993; Rybak 1994; Hammer and Menzel 1995).

nal lobes (e.g., Mobbs 1982). Terminals of the Pel in the protocerebral lobe and $A 4$ band of the MB are swollen bleb-like varicosities.

\section{Discussion}

The Pel neuron is a unique neuron in the central brain of the honeybee. Its impressive size, its particular shape, and its location inspired physiological (Mauelshagen 1993) and anatomical (Rybak 1994) studies. The Pel neuron responds to odor stimuli and changes its response properties as a consequence of sensitizing and conditioning trials (Mauelshagen 1993). Sensitization leads to an increase of subsequent odor responses. A single conditioning trial results in a reduction of the sustained response to the conditioned odor; multiple differential conditioning trials enhance responses to the CS+ (odor stimulus paired with sucrose) but leave response to the CS- (specifically unpaired odor stimulus) unchanged. These associative changes of responsiveness appear not to last long
( 10 min). Thus the Pel neuron implements learned olfactory information transiently.

The extended dendritic arbors of the PE1 neuron within the MB indicate that it might be sensitive to multiple sensory input. We find that all odors tested lead to excitatory responses without any indication of odor-specific response patterns. The recordings were also carefully inspected for any rhythmicity of action potentials. We found that only spontaneous activity may show rhythmic spiking with bursts of two to three APs at $80-200 \mathrm{~Hz}$ separated by intervals in the range of $150-250$ msec. Odor stimuli did not induce any rhythmic spiking. Simultaneous recording of field potentials are necessary to test whether there is any temporal component of the odor-induced response as was found for projection neurons in the antennal lobe of the locust (Laurent and Davidowitz 1994; Wehr and Laurent 1996) and the honeybee (Stopfer et al. 1997). No motor patterns of the antennae or the proboscis could be related to the occurrence of rhythmic spiking during spontaneous activity. In addition to its broad sensitivity to odorants, the Pe1 neuron is also sensitive to other sensory modalities. Visual sensitivity appears to be rather unspecific with respect to the stimuli tested (light $\mathrm{ON}$ and OFF, moving black-and-w hite stripes). How ever, it may well be that certain complex features could lead to much stronger responses. Although we have tried to search for particular stimulus combinations using rather simple forms of stimuli, no particularly effective combinations were found. A combination of olfactory and mechanical stimulation induced stronger responses than the single stimuli, but the variability of the responses did not allow evaluation of whether the responses to the combined stimuli exceeded a simple summation of excitation induced by the single stimuli.

How is the multisensory property of the Pel neuron reflected in its anatomy? The Pel neuron is a MB extrinsic neuron. According to Mobbs (1982) $M B$ extrinsic neurons can be distinguished into broad- and narrow-field neurons. The Pel neuron belongs to a group of broad-field MB extrinsic cells that project extensively within the MB neuropil. Other examples of this type of neurons are the A6 neurons that arborize throughout the whole anterior $\alpha$-lobe (Rybak and Menzel 1993). In contrast, $\alpha$-lobe extrinsic neurons like the A4 cells, occupy relatively small bands in the ventral $\alpha$-lobe (Rybak and Menzel, 1993).

Arborizations in the protocerebral lobe bear mostly sw ollen varicosities, indicating their presyn-

\section{$\begin{array}{llllllllllllllll}L & E & A & R & N & I & N & G & \underset{\mathbf{1 4 2}}{\mathbf{X}} & M & E & M & O & R & Y\end{array}$}


aptic nature. This is supported by preliminary results on the EM level (Rybak and Mauelshagen 1994). Therefore, the existing terminal fields of the Pel in the ring neuropil around the $\alpha$-lobe and in the $\mathrm{LPL}$, including the $\mathrm{LH}$, indicate that the Pel neuron receives most or all input from within the Kenyon cells of the MB. The Pel neuron does not resemble the type of complex $M B$ extrinsic neurons with dendritic and terminal fields outside the $M B$, which supply afferent information to the lobes as described in Periplaneta ( $\mathrm{Li}$ and Strausfeld 1997). However, the Pel forms collaterals that project back into the A4 band of the $\alpha$-lobe and form presumably output synapses here (Rybak and Mauelshagen 1994). The importance of the A4 zone for processing olfactory information is substantiated by the fact that it receives information via the Kenyon cell axons that originate primarily from the lip subcompartment of the calyces (Rybak 1994). Optical recordings using a $\mathrm{Ca}^{2+}$-sensitive dye indicated that odor stimulation elicits activity in the calyces and the A4 zone of the $\alpha$-lobe (J. Joerges, G. Galizia, and R. Menzel, unpubl.). A4 neurons project to the LPL, where they overlap partially with the Pel neuron projections. It thus appears that the multisensory responses of Pel are forwarded onto neurons apparently selectively devoted to olfactory coding both in MBs and LPLS.

The analysis of the dendritic Pel neuron pattern within the MB reveals further important properties of this neuron. The comparison with the internal structure of Kenyon cell projections substantiates the multimodal sensitivity of the Pel neuron (see Fig. 5). The main branch of the Pel neuron enters at the tripartition of the peduncle and both lobes, and gives rise to extensive arborizations throughout the vertically oriented zones of the peduncle (Figs. 5 and 6). Thus, the dendritic arbors of the Pel neuron cover areas in the peduncle that consist of Kenyon cell axons from all calycal subcompartments, as the vertical stripes as a whole represent all calycal subcompartments. Ultrastructural studies confirm that the Pel neuron receives synaptic input from Kenyon cells (Rybak and Mauelshagen 1994). Therefore, the anatomy indicates that the Pel neuron would be ideally suited to integrate information across the sensory modalities reaching the MB. The ordered input into the MB calyx from olfactory, visual, and mechanosensory systems is maintained throughout the peduncle and the lobes by the parallel Kenyon cell bundles with only a few exceptions to this rule (Rybak 1994). For example, Kenyon cells were found that arborize in an unusual manner in both collar and lip subcompartments. Feedback neurons of the protocerebro calycal tract [p.c.t. (Bicker et al. 1985; Gronenberg 1987)], which connect output regions of the MB with the calycal input sites, mix information processed in different compartments of the MB and may also be responsible for the multimodal properties of a variety of extrinsic neurons (for review, see Erber et al. 1987). However, p.c.t. neurons do not innervate the ventral $\alpha$-lobe (to which the A4 zone belongs; $B$. Grünewald, unpubl.). Multisensory integration might therefore mainly be performed by neurons like the Pe1 neuron or A6 neurons.

The presumable output areas of the Pel neuron are in the $\mathrm{LPL}$, mainly in the $\mathrm{LH}$ and in the ring neuropil around the $\alpha$-lobe (Fig. 7). In the LPL, a presumable premotor area (e.g., Li and Strausfeld 1997), it overlaps with neurons of the ACT, the VUMmx 1 neuron (Hammer 1993), and a recurrent neuron from the MB to the antennal lobes, the AL-1 (Rybak and Menzel 1993). The convergence with the modulatory VUM neurons may indicate that the Pel neuron is under the control of presynaptic modulatory action. The convergence with the AL-1 neuron feeding back to the antennal lobe may indicate that output from the MB is provided to the antennal lobe, possibly with the contribution of the Pel neuron, and thus in a relationship to Pel's specific properties. Therefore, we forward the hypothesis that such a contribution may be view ed in the context of memory consolidation.

According to its response properties and its connectivity, the Pel neuron can be incorporated in a hypothetical model of the neural substrate of learning and memory formation in the honeybee brain. Multisensory convergence at the Pel neuron may be a potential neural substrate for contextual coding of olfactory stimuli and may be related to synaptic processes that underlie context-dependent learning of olfactory stimuli. Contextual learning is a well-documented property of honeybee learning under natural conditions (Menzel 1990; Menzel et al. 1996).

The information conveyed by the Pel neuron is multisensory and MB processed. In the shortterm range following learning, the Pel neuron reports about a learned odor possibly in relationship to contextual stimuli. A comparison with the information transmitted directly from the antennal lobe to the LPL via olfactory projection neurons (ACTs in Fig. 7) may lead to a signal that indicates the difference between the information coming from

$$
\begin{array}{llllllllllllllll}
L & E & A & R & N & I & N & G & \underset{143}{\mathbf{X}} & M & E & M & O & R & Y
\end{array}
$$


the antennal lobe and the MB. This signal may indicate the amount of learned information as processed in the MB, thus not only with respect to the olfactory stimulus but also the context in which the odor occurred. Furthermore, such a signal may be evaluated in reference to the information provided by the VUM neurons, because VUM neurons project also to the LPL. The hypothetical signal may be transmitted not only to premotor neurons but via neurons like the AL-1 also back to the antennal lobe. It is conceivable that this signal serves the function of memory consolidation at the level of the antennal lobe and instructs the antennal lobe about the transient learning processes occurring in the Pel. A consequence of this model is that important aspects of olfactory memory including context dependencies may influence the process of memory formation in the antennal lobe under the control of the MB. Such a hypothesis can readily be tested in the bee (see also Hammer and Menzel 1998), because the specific components of memory formation in the antennal lobe and MB are experimentally accessible.

\section{Acknowledgments}

We are most grateful to Dr. B. Grünewald for carefully commenting on earlier versions of the manuscript, Astrid Klawitter for help with the Bodian procedure, and Sybille Schaare and Dr. Robert Brandt for their most valuable help with the figures. The reconstruction of $\mathrm{Pel}$ presented in Figure 1 is based on intracellular marking performed by Janna Klein, whose contribution we gratefully acknowledge. The work presented here was supported by the Sonderforschungsbereich 515.

The publication costs of this article were defrayed in part by payment of page charges. This article must therefore be hereby marked "advertisement" in accordance with 18 USC section 1734 solely to indicate this fact.

\section{References}

Bicker, G., S. Schäfer, and T.G. Kingan. 1985. Mushroom body feedback interneurons in the honeybee show GABA-like immunoreactivity. Brain Res. 360: 394-397.

Blest, A.D. 1961. Some modifications of Holme's silver method for insect nervous system. Q. J. Microsc. Sci. 102: 413-417.

Clark, G. 1973. Neurological staining methods: Bodian's protargol method. In Staining procedures used by the biological stain commission (ed. G. Clatk), pp. 98-100. Williams \& Wilkins, Baltimore, MD.

Davis, R.L. 1993. Mushroom bodies and Drosophila learning. Neuron 11: 1-14. de Belle, J.S. and M. Heisenberg. 1994. Associative odor learning in Drosophila abolished by chemical ablation of mushroom bodies. Science 263: 692-695.

Erber, J., U. Homberg, and W. Gronenberg. 1987. Functional roles of the mushroom bodies in insects In Arthropod brain: Its evolution, development, structure, and functions (ed. A.P. Gupta), pp. 485-511. John Wiley and Sons, New York, NY.

Gronenberg, W. 1987. Anatomical and physiological properties of feedback neurons of the mushroom bodies in the bee brain. Exp. Biol. 46: 115-125.

Hammer, M. 1993. An identified neuron mediates the unconditioned stimulus in associative olfactory learning in honeybees. Nature 366: 59-63.

Hammer, M. and R. Menzel. 1995. Learning and memory in the honeybee. J. Neurosci. 15: 1617-1630.

--- M Multiple sites of associative odor learning as revealed by local injections of octopamine in honeybees. Learn. \& Mem. (this issue).

Heisenberg, M., A. Borst, S. W agner, and D. Byers. 1985. Drosophila mushroom body mutants are deficient in olfactory learning. J. Neurogenet. 2: 1-30.

Homberg, U. 1984. Processing of antennal information in extrinsic mushroom body neurons of the bee brain. J. Comp. Physiol. A 154: 825-836.

Howse, P.E. 1974. Design and function in the insect brain In Experimental analysis of insect behaviour. (ed. L. Barton-Browne), pp. 180-194. Springer, Berlin, Germany.

Kenyon, F.C. 1896. The brain of the bee-A preliminary contribution to the morphology of the nervous system of the Arthropoda. J. Comp. Neurol. 6: 134-210.

Laurent, G. and H. Davidowitz. 1994. Encoding of olfactory information with oscillating neural assemblies. Science 265: 1872-1875.

Li, Y. and N.J. Strausfeld. 1997. Morphology and sensory modality of mushroom body extrinsic neurons in the brain of the cockroach, Periplaneta americana. J. Comp. Neurol. 387: $631-650$.

Mauelshagen, J. 1993. Neural correlates of olfactory learning in an identified neuron in the honey bee brain. $J$. Neurophysiol. 69: 609-625.

Menzel, R. 1990. Learning, memory, and "cognition" in honey bees. In Neurobiology of comparative cognition (ed. R.P. Kesner and D.S. OIten), pp. 237-292. Erlbaum Inc., Hillsdale, NJ.

Menzel, R., J. Erber, and T. Masuhr. 1974. Learning and memory in the honeybee. In Experimental analysis of insect behaviour (ed. L. Barton-Browne), pp. 195-217. Springer, Berlin, Germany. 
Menzel, R., C. Durst, J. Erber, S. Eichmüller, M. Hammer, H. Hildebrandt, J. M auelshagen, U. M üller, H. Rosenboom, J. Rybak, S. Schäfer, and A. Scheidler. 1994. The mushroom bodies in the honeybee: From molecules to behavior. Neural basis of behavioral adaptations. Fortschr. Zool. 39 81-102.

Menzel, R., K. Geiger, L. Chittka, J. Joerges, J. Kunze, and U. Müller. 1996. The knowledge base of bee navigation. J. Exp. Biol. 199: 141-146.

Mobbs, P.G. 1982. The brain of the honeybee Apis mellifera I. The connections and spatial organization of the mushroom bodies. Philos. Trans. R. Soc. Lond. B Biol. Sci.

298: 309-354.

Rybak, J. 1994. “Die strukturelle O rganisation der Pilzkörper und synaptische Konnektivität protocerebraler Interneuronen im Gehirn der Honigbiene, Apis mellifera. Eine licht- und elektronenmikroskopische Studie", Ph.D. dissertation. Freie U niversität Berlin, Germany.

Rybak, J. and R. Menzel. 1993. Anatomy of the mushroom bodies in the honey bee brain: The neuronal connections of the alpha-lobe. J. Comp. Neurol. 334: 444-465.

Rybak, J. and J. Mauelshagen. 1994. The Pe1-neuron of the honeybee-An afferent pathway from the mushroom bodies to the protocerebral lobe. In Proceeding of the

Twenty-second Göttingen Neurobiology Conference Vol. II, p. 830. Thieme Verlag, Stuttgart, Germany.

Schürmann, F.W. 1974. Bemerkungen zur Funktion der Corpora pedunculata im Gehirn der Insekten aus morphologischer Sicht. Exp. Brain Res. 19: 406-432.

Stopfer, M., S. Bhagavan, B.H. Smith, and G. Laurent. 1997. Impaired odour discrimination on desynchronization of odour-encoding neural assemblies. Nature 390: 70-74.

Strausfeld, N.J. 1980. The Golgi method: Its application to the insect nervous system and the phenomen of stochastic impregnation. In Neuroanatomical techniques. Insect nervous system (ed. N.J. Strausfeld and T.A. Miller), pp. 131-203. Springer Verlag, Berlin, Germany.

Vowles, D.M. 1955. The structure and connections of the corpora pendunculata in bees and ants. Q. J. Microsc. Sci. 96: $239-255$.

Wehr, M. and G. Laurent. 1996. O dour encoding by temporal sequences of firing in oscillating neural assemblies. Nature 384: 162-166.

Witthöft, W. 1967. Absolute Anzahl und Verteilung der Zellen im Hirn der Honigbiene. Z. Morph. Tiere

61: $160-184$.

Received January 28, 1998; accepted in revised form May 11, 1998. 


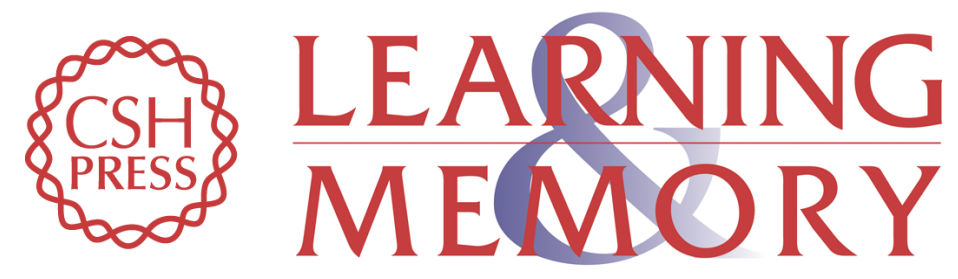

\section{Integrative Properties of the Pe1 Neuron, a Unique Mushroom Body Output Neuron}

Jürgen Rybak and Randolf Menzel

Learn. Mem. 1998, 5:

Access the most recent version at doi:10.1101//m.5.1.133

References This article cites 22 articles, 4 of which can be accessed free at: http://learnmem.cshlp.org/content/5/1/133.full.html\#ref-list-1

License

Email Alerting Receive free email alerts when new articles cite this article - sign up in the box at the Service top right corner of the article or click here. 